\title{
Aspectos semánticos de la polisemia somática en el quechua Ayacucho-chanca: los casos de uma 'cabeza' y simi 'boca'
}

\author{
Semantic aspects of somatic polysemy in the quechua Ayacucho- chanca : the \\ cases of uma 'head' and simi ' mouth' \\ Isabel Gálvez Astorayme ${ }^{a}$, Isabel Judith Gálvez Gálvez ${ }^{a}$, Frank Joseph Domínguez \\ Chenguayen $^{a}$ \\ ${ }^{a}$ Universidad Nacional Mayor de San Marcos \\ Contacto: igalveza@unmsm.edu.pe
}

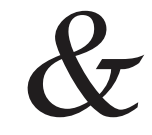

Resumen

Los lexemas referidos a la anatomía humana en el quechua Ayacuchochanca transgreden el significado literal (o convencional) y constituyen unidades fuertemente polisémicas en la lengua y cultura quechua. En el presente artículo, analizamos los lexemas somáticos uma 'cabeza' y simi 'boca', los cuales evidencian un complejo universo conceptual y forman una red semántica provista de una estructura radial interna. En particular, nuestros objetivos son dos. Por una parte, nos interesa identificar todos aquellos conceptos a los que estas dos formas linguísticas, referidas a la biología humana del quechuahablante, están asociadas. Por otra parte, pretendemos explicar la manera en que estos nuevos conceptos habrían llegado a asociarse a los referidos lexemas somáticos. Para ello, nos basaremos en los principios teóricos y metodológicos de la disciplina denominada linguiística cognitiva (Lakoff y Johnson 1980, Langacker 1987, Lakoff 1987, entre otros), y propondremos la metáfora y la metonimia como aquellos mecanismos responsables de la extensión de los significados.

Palabras claves: Linguiística cognitiva, polisemia, quechua, lexemas somáticos.

\footnotetext{
${ }^{1}$ El artículo que presentamos formó parte del proyecto de investigación Aspectos semánticos de la polisemia en los somatismos del quechua ayacuchano, el cual fue presentado al Instituto de Investigaciones Lingüísticas (INVEL) y desarrollado durante el año 2015. Esta investigación fue dirigida por Dra. Isabel Gálvez Astorayme, y en ella participaron además de los autores, Franklin Espinoza Bustamante y el egresado Óscar Huamán Águila. Asimismo, queremos agradecer la especial participación de Franklin Espinoza Bustamante y de Óscar Huamán Águila en la recopilación del corpus durante el desarrollo del proyecto.
} 
Isabel Gálvez Astorayme, Isabel Judith Gálvez Gálvez, Frank Joseph Domínguez Chenguayen

\begin{abstract}
Lexemes related to human anatomy in Quechua Ayacucho-Chanca transgress the literal (or conventional) meaning, go beyond it, and are strongly polysemic units in the Quechua language and culture. In this research, we analyze the somatic lexemes uma 'head' and simi 'mouth', which show a complex conceptual universe, and form a semantic network provided with an internal radial structure. In particular, our goals are twofold. On the one hand, we want to identify all those concepts that these two linguistic forms related to human biology Quechua, is associated. Moreover, we intend to explain how these new concepts have come to be associated with somatic lexemes mentioned before. To do this, we will build on the theoretical and methodological principles of the discipline called cognitive linguistics (Lakoff and Johnson 1980, Langacker 1987, Lakoff 1987, among others), and propose the metaphor and metonymy as those mechanisms responsible for the extension of meanings.

Keywords: Cognitive linguistics, Polysemy, Quechua, Human body terms.
\end{abstract}

Recibido: 12/5/16.

Aceptado: 18/6/16

\title{
1. Introducción
}

Los lexemas referidos a la biología humana en el quechua Ayacuchochanca muestran un comportamiento semántico que trasciende el significado lingüístico convencional y configura un universo conceptual único y diverso en la lengua y cultura del quechuahablante. En la región topográfica de la cabeza, tenemos, por ejemplo, los siguientes casos:

(1)

a. Umasapa

Sig. lit.: 'De cabeza grande' Sig. fig.: 'De gran inteligencia'

c. Titirapa sinqan

Sig. lit.: 'De la tetera su nariz' Sig. fig.: 'El pico de la tetera'

e. Llaqtapa rinrin

Sig. lit.: 'Del pueblo su oreja' Sig. fig.: 'El oído del pueblo'

g. Iskay uya

Sig. lit.: 'Dos caras'

Sig. fig.: 'Dos actitudes, hipócrita' b. Papapa ñawin

Sig. lit.: 'De la papa su ojo' Sig. fig.: 'La yema de la papa'

d. Nina qallu

Sig. lit.: 'Lengua de fuego'

f. Nanaq simi

Sig. lit.: 'Hiriente boca' Sig. fig.: 'De habla ofensiva'

Letras 87(126), 2016 
Los lexemas somáticos uma 'cabeza', ñawi 'ojo', sinqa 'nariz', qallu 'lengua', rinri 'oreja', simi 'boca' y uya 'cara' revelan conceptualizaciones diversas, tales como inteligencia (1a), yema (1b), pico (1c), habla (1d y 1f), oído (1e) y actitud (1g).

Los lexemas somáticos presentes en la región topográfica del tronco manifiestan el mismo comportamiento:

(2)
a. Chiri sunqu
b. Uru siki
Sig. lit.: 'Frío corazón' Sig. fig.: 'De sentimiento'
Sig. lit.: 'Gusano trasero' Sig. fig.: 'Persona andariega'
c. Wasachay
d. Wisllapa chupan
Sig. lit.: 'Hacer espalda' Sig. fig.: 'dar apoyo, apoyar'
Sig. lit.: 'Del cucharón su cola' Sig. fig.: 'El mango del cucharón'

e. Matipa pupun

Sig. lit.: 'Del mate su ombligo'

Sig. fig.: 'Punto central del mate'

En estos ejemplos, los somatismos sunqu 'corazón', siki 'trasero', wasa 'espalda', chupa 'cola' y pupu 'ombligo' señalan los sentidos de sentimiento, persona, apoyo, mango y parte central, respectivamente.

Lo mismo sucede, aunque en menor índice, con los lexemas somáticos inscritos en la región topográfica de las extremidades, cuyos sentidos aluden a los conceptos de control (3a) y soporte (3b):

(3)

a. Wayra maki

Sig. lit.: 'Viento mano'

Sig. fig.: 'Que no tiene control de los objetos que posee, derrochador'

\section{b. Misapa chakin}

Sig. lit.: 'De la mesa su pie' Sig. fig.: 'El soporte de la mesa'

Como se ha visto, estas expresiones linguiísticas constituyen la puerta de entrada al sistema conceptual del nativohablante de quechua ayacuchano; sin embargo, de todos los somatismos presentados, uma 'cabeza' y simi 'boca' constituyen dos de los lexemas somáticos, como veremos en (4) y en (5), respectivamente, con mayor índice de expresiones que transgreden el lenguaje 
literal, y configuran un complejo abanico de posibles conceptos:

(4)

a. Tullu uma

Sig. lit.: 'Tronco cabeza'

Sig. fig.: 'Que carece de inteligencia'

c. Chakrapa uman

Sig. lit.: 'De la chacra su cabeza'

Sig. fig.: 'Parte superior de la chacra'

e. Uma hampiy

Sig. lit.: 'Cabeza curar'

Sig. fig.: 'Recuperar la sobriedad'

g. Wayra uma

Sig. lit.: 'Viento cabeza'

Sig. fig.: 'Memoria de viento, olvidadizo'

i. Allin umayuq

Sig. lit.: 'Buena cabeza'

Sig. fig.: 'Que tiene buen pensamiento'

(5)

\section{a. Chiki simi}

Sig. lit.: 'Malagüero boca' Sig. fig.: 'Boca salada'

c. Simisapa

Sig. lit.: 'Grande boca' Sig. fig.: 'Chismoso'

e. Nanaq simi

Sig. lit.: 'Hiriente boca'

Sig. fig.: 'De habla ofensiva'

g. Simin simin puriy

Sig. lit.: 'De boca en boca andar' Sig. fig.: 'Estar en boca de todos'

i. Urqupa simin

Sig. lit.: 'Del cerro su boca' b. Uma kay

Sig. lit.: 'Ser cabeza'

Sig. fig.: 'El que dirige'

d. Uma chinkay

Sig. lit.: 'Cabeza perder'

Sig. fig.: 'Perder la cordura'

f. Umapi apay

Sig. lit.: 'En la cabeza llevar'

Sig. fig.: 'Llevar en el pensamiento'

h. Huk umalla

Sig. lit.: 'Una sola cabeza'

Sig. fig.: 'Una misma idea'

j. Yana uma

Sig. lit.: 'Negro cabeza'

Sig. fig.: 'De actitud negativa' b. Chuya simi

Sig. lit.: 'Limpia boca'

Sig. fig.: 'De buena educación'

d. Simi apaq

Sig. lit.: 'Boca el que lleva'

Sig. fig.: 'Correveidile'

f. Simi wichqay

Sig. lit.: 'Boca cerrar'

Sig. fig.: 'Guardar el secreto'

h. Mankapa simin

Sig. lit.: 'De la olla su boca' Sig. fig.: 'Abertura de la olla'

j. Mana simiyuq

Sig. lit.: 'Sin boca' 
A la luz de estos datos, y otros que iremos presentando, los objetivos de nuestro trabajo son los siguientes: a) identificar todos aquellos posibles conceptos (o sentidos) asociados a las formas quechuas uma 'cabeza' y simi 'boca'; es decir, nos interesa evaluar el alcance semántico que muestran los referidos lexemas somáticos en la lengua y cultura quechua; b) explicar la manera en que todos aquellos potenciales sentidos habrían llegado a asociarse a las referidas formas linguísticas; esto es, establecer los mecanismos que han permitido la construcción de nuevos sentidos. Para ello, nos basaremos en los principios teóricos y metodológicos de la linguiística cognitiva (Lakoff 1987, Langacker 1987, Talmy 2000, entre otros). Esta corriente teórica se explica en $§ 2$. En §3, analizamos la semántica de los lexemas somáticos uma 'cabeza' y simi 'boca'. Finalmente, se exponen las conclusiones.

\section{La polisemia en el marco de la lingüística cognitiva}

La polisemia en el seno de la linguística cognitiva se define inicialmente apelando al concepto de unidad simbólica (cf. Langacker 2008). A diferencia de otros fenómenos como la homonimia y la sinonimia, se caracteriza por presentar dos conceptos (o más) bajo una sola forma.

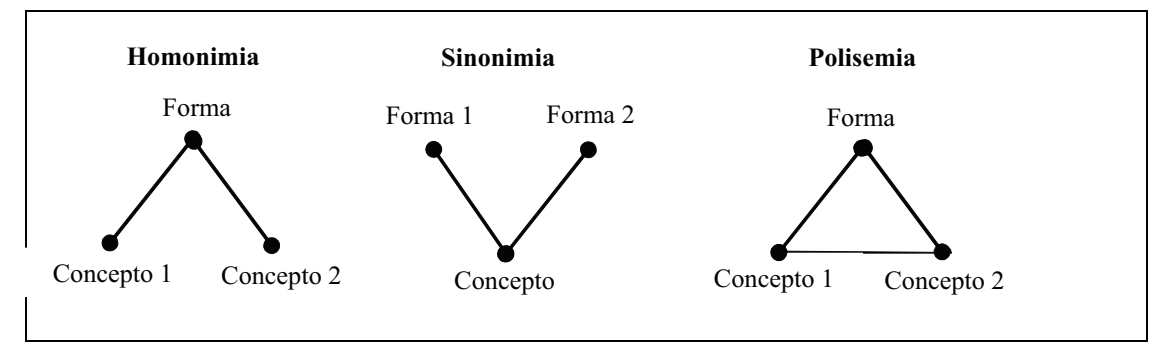

Figura 1. Diferencias entre homonimia, sinonimia y polisemia

En efecto, si los sinónimos se conciben como la expresión de un (mismo) concepto bajo dos formas diferentes, entonces las palabras polisémicas reúnen dos conceptos diferentes -relacionados entre sí- bajo una misma forma, 
a diferencia de los términos homónimos. Tras esa distinción, lo que sigue sería averiguar cómo están organizados dichos conceptos, la manera en que llegaron a generarse y el modo en que potencialmente pueden representarse en el sistema linguiístico. Las secciones que vienen dan cuenta de la teoría que permite explicar en detalle este fenómeno.

\subsection{La polisemia como categorización}

Desde el enfoque de la linguística cognitiva, la polisemia ha sido vista como un proceso de categorización (Lewandowska-Tomaszczyk 2007), lo cual implica asumir, respecto de los conceptos (o sentidos), un elemento prototípico y una serie de elementos periféricos (o no prototípicos). En otras palabras, todo lexema polisémico presenta un significado central y un conjunto de significados menos centrales (o más periféricos). De este modo, como señalan Evans y Green (2006: 333), es posible hacer las siguientes observaciones:

a) Las palabras y sus sentidos representan categorías conceptuales, las cuales tienen mucho en común con las categorías conceptuales no linguiísticas. De allí se sigue que las categorías linguiísticas tienen estructura prototípica.

b) Los significados de la palabra son típicamente polisémicos; se estructuran respecto de un prototipo central. Las categorías léxicas forman entonces categorías radiales, las cuales pueden ser modeladas como estructuras de irradiación.

c) Las categorías radiales, particularmente las extensiones de significado a partir del prototipo, se encuentran motivadas por mecanismos cognitivos.

d) Los sentidos que constituyen categorías radiales se encuentran almacenados en una red esquemática.

\subsection{Procedimientos cognitivos de extensión semántica}

Si en la sección anterior quedó claro que los múltiples significados de una palabra polisémica no tienen el mismo estatus, esto es, que uno es el central y los demás son periféricos, entonces de lo que se trata ahora es de ver qué teoría puede dar cuenta de la extensión de esos elementos no centrales. Para ello, Lakoff y Johnson (1980), Lakoff (1987), entre otros, han propuesto que el origen de los sentidos pueden explicarse por procesos cognitivos de naturaleza metafórica y metonímica. 
La metáfora, entendida en los términos A ES B, da cuenta del surgimiento de un nuevo concepto A apelando a otro ya existente B. Por ejemplo, si quisiéramos explicar por qué en la expresión ya veo lo que quieres decir se presenta un nuevo sentido en el verbo ver diferente a 'percibir visualmente algo', tendríamos que recurrir a la metáfora COMPRENDER ES VER, donde se pone de manifiesto un concepto en términos de otro mediante un proceso de correlación experiencial entre la percepción visual que alcanza el ojo humano y la comprensión inmediata que ello supone de la realidad. Por esa razón, es natural que la metáfora, en tanto correlacione situaciones en el devenir cotidiano, conduzca la asociación de dos conceptos diferentes y, con ella, la adhesión de uno de ellos a una misma forma lingǘstica. Por otro lado, la metonimia se presenta bajo el formato A POR B, y, a diferencia de la metáfora, el dominio fuente no lo constituye B, sino A. Un nuevo sentido B es explicable así mediante otro A. Por ejemplo, en la expresión ese hombre no tiene corazón se explica el surgimiento del concepto de sentimiento por medio de la metonimia EL CORAZÓN POR LOS SENTIMIENTOS. De esta manera, tanto las metonimias como las metáforas constituyen mecanismos (o procedimientos) cognitivos de extensión semántica que permiten asociar nuevos conceptos a una forma linguística ya establecida.

Por convención tipográfica, las metáforas son señaladas mediante versalitas, mientras que las expresiones metafóricas, consecuencia de aquellas, en itálicas.

\subsection{La red semántica}

La red semántica constituye el aparato formal que permite identificar radialmente cuántos sentidos están asociados a una forma lingüística. Existen varios modelos que intentan dar cuenta de ello y del estatus teórico que estas

\footnotetext{
${ }^{4}$ Por convención tipográfica, las metáforas son señaladas mediante versalitas, mientras que las expresiones metafóricas, consecuencia de aquellas, en itálicas.
} 
redes semánticas representan en la estructura conceptual de los hablantes. Nosotros asumiremos la observación de Tyler y Evans (2003: 39): «A model is only useful to the extent that it provides a reasonable approximation of how language users might mentally represent the semantic network associated with a particular form». En seguida, presentamos el esquema radial que asumiremos.

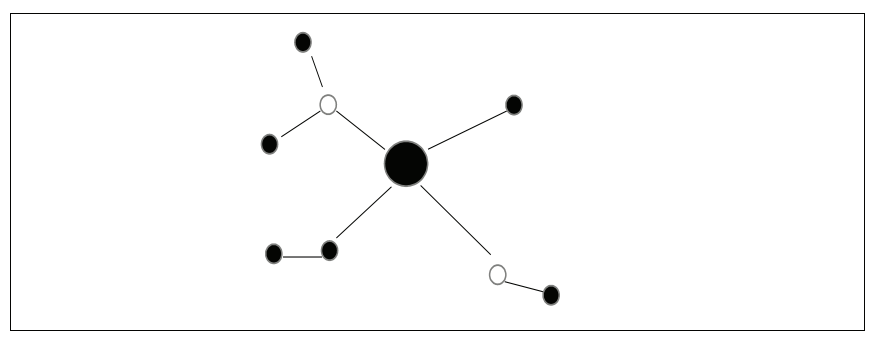

Figura 2. Red Semántica (adaptada de Evans y Green, 2006, p. 332)

El esquema formaliza la representación radial de una unidad simbólica polisémica asociada a un conjunto de sentidos. Estos sentidos irradian de un nódulo o centro (de allí el nombre de significado central y significados no centrales o periféricos). Asimismo, es probable encontrar conjuntos de sentidos que estén organizados por cierto tipo de afinidades.

3. Aspectos semánticos de la polisemia somática en el quechua Ayacucho-chanca

Describimos y explicamos los aspectos semánticos de los lexemas somáticos uma 'cabeza' y simi 'boca'. Entendemos por aspectos semánticos todas aquellas conceptualizaciones a las que los referidos términos están sujetos. La evidencia que presentamos constituye un conjunto de expresiones linguísticas que respaldan el complejo universo conceptual que manifiesta la lengua y cultura quechua.

${ }^{5}$ Nuestra traducción: «Un modelo es únicamente útil en el sentido de que provea una aproximación razonable de cómo los usuarios podrían mentalmente representar la red semántica asociada a una forma particular». 


\section{1. La semántica del lexema somático uma 'cabeza'}

En este apartado, el lexema somático uma es analizado respecto del complejo abanico conceptual que manifiesta en el sistema lingǘstico quechua.

\subsubsection{Más allá del significado lingüístico convencional}

Los significados que trascienden los significados lingüísticos tradicionales constituyen extensiones semánticas no prototípicas (o periféricas). Como evidenciaremos, estos sentidos representan un complejo banco léxico que forja una memoria semántica única respecto del lexema en cuestión para el quechuahablante.

\subsubsection{Sentidos relacionados en torno al eje de verticalidad}

Los sentidos que se analizan están referidos al eje de verticalidad, eje que permite, como detallaremos más adelante, ordenarlos y darles origen.

\subsection{Uma 'jefe'}

Un primer sentido de uma alude a la persona que tiene el mando, el control o la que dirige a otros. La siguiente expresión corrobora el concepto:

(1) Runapa uman

Sig. fig.: 'El que dirige a la gente'

El lexema uma ya no es evocado en la lengua y cultura del quechuahablante como 'cabeza', sino como 'jefe'. Como se evidencia, el quechuahablante utiliza una relación metonímica y accede al concepto LA PERSONA QUE DIRIGE, a través de otro concepto, LA REGIÓN TOPOGRÁFICA SUPERIOR DEL CUERPO HUMANO. De este modo, esta metonimia puede ser reactivada en diferentes contextos lingüísticos, pero siempre bajo la misma forma linguística.

(2) Pedro maqtaqa allin umallikuqmi.

Sig. fig.: 'Pedro es un buen dirigente'.

Un requisito ineludible para que aparezca este sentido, independientemente de las formas gramaticales que lo acompañan (e. g., umallikuq 'el que dirige'), es la presencia del referido lexema de la anatomía humana. De esta manera, y con el uso constante, este sentido se configura en la cultura quechua. 
(3) Allin umallikuqwan yakuta mañakamusunchik chakranchikpaq.

Sig. fig.: 'Con un buen dirigente, pidamos agua para nuestra chacra'.

\subsection{Uma 'parte superior'}

Este sentido se utiliza como un referente espacial, y alude a la parte de superior de alguna entidad. La expresión siguiente deja evidencia de este nuevo concepto:

(4) Chakrapa umanpim punku kachkan.

Sig. fig.: 'En la parte superior (cabecera) de la chacra está la puerta'.

En este caso, la cabeza de la chacra se entiende como la cabecera de aquella (i. e., la parte superior), lo cual se explica mediante la metonimia LA REGIÓN TOPOGRÁFICA SUPERIOR DEL CUERPO HUMANO POR LA PARTE DE ARRIBA.

(5) Sachapa umampin paka paka wawachkan.

Sig. fig.: 'En la parte superior del árbol (cabecera) está cantando la lechuza'.

En este caso, el referente, sujeto a la conceptualización de uma, ya no es la chacra, sino sacha 'árbol'. No obstante, el referente espacial en la nueva conceptualización sigue siendo el mismo: la parte superior de alguna entidad.

\subsubsection{Sentidos relacionados a la cognición humana}

En esta sección, se analizan aquellos sentidos que tienen que ver con la cognición o que participan en relación a ella.

\subsection{Uma 'inteligencia'}

Esta nueva conceptualización alude a la capacidad de adaptarse a nuevas situaciones; esto es, al concepto de inteligencia. Observemos la expresión siguiente:

(6) Tayta Andresqa umasapam, chaymi achka yachayniyuq.

Sig. fig.: 'El señor Andrés es inteligente y por eso tiene muchos conocimientos'.

La metonimia que caracteriza el surgimiento de este nuevo sentido reside en LA REGIÓN TOPOGRÁFICA SUPERIOR DEL CUERPO POR LA INTELIGENCIA. Este es un hecho esperable si consideramos que existe cierto tipo de conciencia de lo 
que sucede en dicha región por parte de los hablantes. Este sentido es así, mediante la referida metonimia, evocado en otros escenarios lingüísticos.

(7) Churinkunapas umasapam.

Sig. fig.: 'Sus hijos son también inteligentes'.

$\mathrm{Si}$ bien es cierto que la cabeza permite inducir un nuevo concepto, el de inteligencia, nótese la presencia de la metáfora para concretar esta nueva conceptualización. La metáfora aparece mediante el sufijo aumentativo-sapa, y es posible comprender gracias a él, la inteligencia en términos dimensionales; por ello, el que tiene grande la cabeza (i. e., umasapa) es el que tiene gran inteligencia.

\subsection{Uma 'pensamiento'}

Este sentido se refiere a la capacidad o facultad de pensar y está contenida en expresiones como las siguientes:

(8) Llaqtaypi llapa yachaykunamanta umaypim apani.

Sig. fig.: 'Los saberes de mi pueblo llevo en mi pensamiento'.

El concepto de pensamiento se obtiene a partir del concepto de cabeza; es decir, se formula la metonimia LA REGIÓN TOPOGRÁFICA SUPERIOR DEL CUERPO HUMANO POR EL PENSAMIENTO.

(9) Huk umalla yachay wasita ruwasun.

Sig. fig.: 'Con un solo pensamiento construyamos la escuela'.

(10) Huk umalla gobiernuta posta medicata mañakamusunchik.

ig. fig.: 'Con un solo pensamiento pidamos una posta médica al gobierno'.

De esta manera, para el quechuahablante es posible referirse al pensamiento mediante el uso del concepto cabeza.

\subsection{Uma 'memoria'}

Esta nueva conceptualización en el lexema alude a la facultad que tiene el quechuahablante para recordar. Evidencia de este nuevo concepto se muestra a continuación:

(11) Wayra uma 
Sig. fig.: 'Memoria frágil, olvidadizo'

En este caso, el concepto de cabeza es utilizado por el quechuahablante para generar el de memoria, pero nótese que este concepto es semánticamente reforzado por medio del lexema wayra 'viento', pues la facultad para recordar tiende a ser una capacidad limitada y, justamente, el referido término permite llevar a cabo dicha caracterización.

(12) Wak wayra uma warmi llikllanta saqirqun.

Sig. fig.: 'Esa mujer olvidadiza dejó su manta'.

(13) Wak maqta wayra uma mamanta yuyanchu.

Sig. fig.: 'Ese muchacho olvidadizo no se acuerda de su madre'.

\subsubsection{Sentidos relacionados a la conducta humana}

Este grupo está relacionado a una serie de conceptos que tienen que ver con el comportamiento que manifiesta el quechuahablante ante cierto tipo de situaciones. En seguida, pasamos a dar cuenta de cada uno de los sentidos inscritos en este grupo.

\subsection{Uma 'cordura'}

Esta nueva conceptualización hace referencia a la característica que evidencia una persona respecto de la prudencia y la sensatez. Sin embargo, la expresión de este nuevo concepto en la lengua y cultura quechua se manifiesta como un atributo ausente:

(14) Uma chinkay.

Sig. fig.: 'Perder la cordura'.

La expresión de esta nueva conceptualización en el lexema somático uma aparecerá en contextos lingüísticos que aluden a circunstancias negativas. Por ejemplo:

(15) Maqta mana allin warmiraykum uma chinkaywan.

Sig. fig.: 'El muchacho por culpa de la mala mujer perdió la cordura'. Como se aprecia, perder la cabeza entraña perder la prudencia y la sensatez, y el sujeto que pierde así la región topográfica superior del cuerpo humano es aquel 
que perdió la cordura.

\subsection{Uma 'sobriedad'}

Este nuevo sentido se refiere al concepto de sobriedad, el que aparece en contextos en los que se destaca el malestar de las personas que beben alcohol e intentan recuperar la sobriedad; dicha sobriedad es aludida mediante el ítem léxico cabeza. Por ejemplo:

(16) Uwihankunata hirrakuspam achka aqawan macharqum, chaymi uma hampiyta munan.

Sig. fig.: 'Haciendo la herranza de sus ovejas se mareó con mucha chicha, por eso, piensa recuperar la sobriedad'.

(17) Warmi aqata ruwaspam macharqun, chaymanta uma hampiyta munan.

Sig. fig.: 'La mujer preparando la chicha se mareó y por eso quiere recuperar la sobriedad'.

En ambos casos se tiene que curar la cabeza presupone recuperar la sobriedad. El ítem léxico cabeza es empleado en el razonamiento del quechuahablante para la creación del nuevo concepto de sobriedad.

\subsection{Uma 'ladrón'}

El sentido de ladrón pone de manifiesto a la persona que comete un acto delictivo. Por ejemplo:

(18) Urqupis yana uma llamakunata suwachkan.

Sig. fig.: 'Dicen que en las punas el ladrón está robando llamas'.

En este caso, el acto delictivo es configurado metafóricamente en términos del concepto al que alude el ítem léxico yana 'negro'. Sin embargo, el ladrón es referido metonímicamente mediante el lexema uma 'cabeza'. Esto da pie a que se generen así otras expresiones y, con ellas, la presencia del nuevo concepto de ladrón.

(19) Kananqa yana umakunas wasikunam yaykuchkan.

Sig. fig.: 'Dicen que ahora los ladrones están entrando a las casas'.

\subsection{Uma 'problema'}

En este nuevo caso, el lexema somático evidencia el concepto de 
problema. La expresión siguiente permite constatar ello:

(20) Mama Juanapa maqtan uma nanayninmi.

Sig. fig.: 'El hijo de doña Juana es su problema'.

La aparición de esta nueva conceptualización se patentiza en la lengua quechua mediante la expresión uma nanay 'cabeza doler'. Para el quechuahablante, el problema se configura metafóricamente mediante la sensación de dolor, pero es referido, sin embargo, metonímicamente por medio del lexema uma; es decir, se configura la relación metonímica LA REGIÓN TOPOGRÁFICA SUPERIOR DEL CUERPO HUMANO POR EL PROBLEMA. Gracias a este razonamiento, otros escenarios lingüísticos son posibles.

(21) Machasqa runakuna llaqtapa uma nanayninmi

Sig. fig.: 'Los alcohólicos son un problema para el pueblo'.

\subsection{Uma 'sentimiento'}

Uma refiere al concepto de sentimiento. La metonimia empleada por el quechuahablante reside en LA REGIÓN TOPOGRÁFICA SUPERIOR DEL CUERPO HUMANO POR EL SENTIMIENTO, y es lo que permite el desarrollo de expresiones como las siguientes:

(22) Tayta Andresqa allin umayuqmi.

Sig. fig.: 'El señor Andrés es de buenos sentimientos'.

Ser de buena cabeza implica así ser de buenos sentimientos. Por esa razón, decir que el señor Andres es de buena cabeza se entiende que tenga buenos sentimientos. A continuación, mostramos un ejemplo más que permite sostener esta nueva conceptualización.

(23) Uwiha michipakuq warmichaqa allin umayuqmi chaymi paymanu wihaykita saqiykuwaq.

Sig. fig.: 'La pastorita tiene buenos sentimientos y es de confianza; por eso, debes dejarle tus ovejas a ella'.

3.1.2. Red semántica asociada al lexema somático uma 'cabeza' 


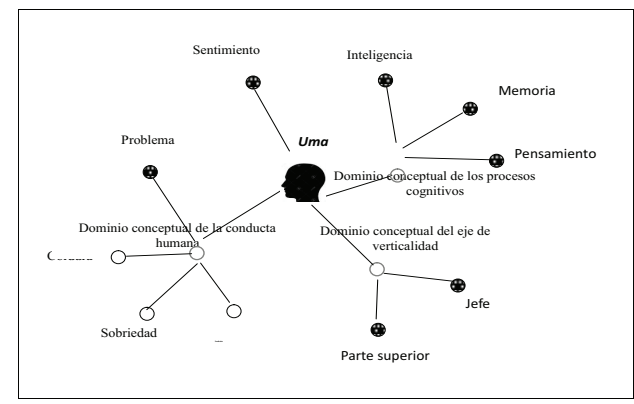

Figura 3. La red semántica del lexema somático uma 'cabeza'

Como puede apreciarse en la red provista, el significado central lo constituye la región topográfica superior de la anatomía del quechuahablante (i. e., uma 'cabeza'). A partir de este significado, es posible ir derivando algunas posibles extensiones semánticas. Estos significados periféricos (o no prototípicos) están organizados del modo siguiente: en el primer grupo (dominio conceptual del eje de verticalidad), se encuentran situados dos sentidos: parte superior y jefe; el segundo grupo (dominio conceptual de los procesos cognitivos) reúne los sentidos de inteligencia, memoria y pensamiento; el tercer grupo (el dominio conceptual de la conducta humana) presenta los sentidos de problema, cordura, sobriedad y ladrón. Por último, uma es utilizado para expresar la noción de sentimiento.

\subsection{La semántica del lexema somático simi 'boca'}

En este apartado, describimos y analizamos cada uno de los sentidos no literales que es capaz de expresar simi 'cabeza' en el quechua Ayacuchochanca.

\subsubsection{Más allá del significado lingüístico convencional}

Los sentidos que trascienden el significado lingǘstico convencional constituyen aquellos significados no prototípicos (o periféricos). Este lexema no tiene el mismo alcance semántico que presenta uma, pero presenta una cantidad mayor de expresiones que facilitan la emergencia de cada uno de los nuevos conceptos a los que la forma linguística simi 'boca' está asociada. 


\subsubsection{Simi 'idioma'}

Mediante la metonimia LA CAVIDAD BUCAL POR EL IDIOMA, el quechuahablante realiza un nuevo concepto, el de lengua (o idioma). Consideremos, por ejemplo, el enunciado siguiente:

(24) Peruano runakuna llapanchik runasimita yachanchikman

Sig. fig.: 'Todos los peruanos debemos aprender el idioma quechua'. Como se observa en la expresión, el término runasimi se entiende como idioma que, debido al contexto, es comprendido por el quechuahablante como su lengua originaria. Esta misma interpretación se produce en otro escenario linguístico.

(25) Peruano runakunapas llapanchik runasimitapas yachanchikman.

Sig. fig.: 'También todos los peruanos debemos aprender aimara'.

En este caso, el término runasimita ya no es interpretado como la lengua quechua, sino como la lengua aimara. Lo que determina la especificidad del idioma es el contexto, pero el referido término sigue expresando la misma idea: la noción de idioma.

\subsubsection{Simi 'habla'}

Mediante la metonimia LA CAVIDAD BUCAL POR EL HABLA, el quechuahablante expresa este nuevo concepto de habla. Evidencia de esta nueva conceptualización se muestra a continuación.

(26) ¿Imatataq rimanki chay chiki simiykiwan?

Sig. fig.: '¿Qué cosa hablas con tu boca (habla) salada?'

(27) Kinraytam richkan simi apaq.

Sig. fig.: 'Por allá va el de habla chismosa'.

En estos casos, el lexema somático simi es utilizado para expresar el concepto de habla. Nótese, sin embargo, que mientras en (26) el concepto es reforzado por el ítem léxico chiki 'salado', en (27) dicho concepto es realzado por el verbo apay 'llevar'. De este modo, la facultad del habla del quechuahablante es caracterizada en una expresión como boca salada, mientras que en la otra, como el que lleva el habla. Esto último supone, asimismo, que el habla se 
conciba como un objeto, el cual es pasible de llevarse (o transportarse). El mismo sentido aparece, incluso, en otras construcciones:

(28) Yachachiq runaqa chuya simiyuqmi.

Sig. fig.: 'El profesor es una persona educada'.

(29) Wak runaqa nanaq simiyuqmi.

Sig. fig.: 'Esa persona es de palabras hirientes'.

En (28), la educación del profesor se refleja en su habla; es decir, lo que permite referirlo como una persona educada es la expresión chuya simi 'de habla limpia, pulcra'. Metafóricamente, tendríamos, además para este caso, UNA PERSONA EDUCADA ES UNA PERSONA DE HABLA PULCRA. En (29), metafóricamente tendríamos también un patrón similar: UNA PERSONA HIRIENTE ES UNA PERSONA DE HABLA QUE HACE DOLER. Así, las expresiones revelan la emergencia de una conceptualización novedosa en la cosmovisión del quechuahablante.

\subsubsection{Simi 'secreto'}

Esta nueva conceptualización es posible debido a la metonimia LA CAVIDAD BUCAL POR EL SECRETO.

(30) Ima willakusqaymanta simiykita wichqaykuy.

Sig. fig.: 'Guarda el secreto de las cosas que te conté.'

Metafóricamente, es posible establecer la relación siguiente: EL QUE GUARDA LA BOCA ES EL QUE GUARDA EL SECRETO. Así se produce la manifestación lingüística de este nuevo concepto en contextos lingüísticos diferentes.

(31) Simikiyta wichqaptiyqa allinmi kasunchik.

Sig. fig.: 'Si guardas el secreto estaremos bien'.

Como se nota, simi ya no alude a la cavidad bucal, sino al conocimiento que se tiene de algo, pero de manera subrepticia (i. e., el secreto).

\subsubsection{Simi 'abertura'}

La metonimia a la que recurre el quechuahablante reside en la relación LA CAVIDAD BUCAL POR LA ABERTURA QUE ESTA PRESENTA. Los ejemplos son los siguientes: 
(32) Mankapa siminqa uyru uyru kasqa.

Sig. fig.: 'La abertura de la olla era muy redonda'.

(33) Urqupa siminpis pichqa runakuna chinkakun.

Sig. fig.: 'Dicen que en la abertura del cerro desaparecieron cinco los hombres'.

En (32), por boca se entiende la abertura (o entrada) que tiene la olla. El concepto se asocia a la forma simi. Lo mismo sucede en (33), pues la abertura que este presenta es referido al término somático.

3.2.2. La red semántica asociada al lexema somático simi 'boca'

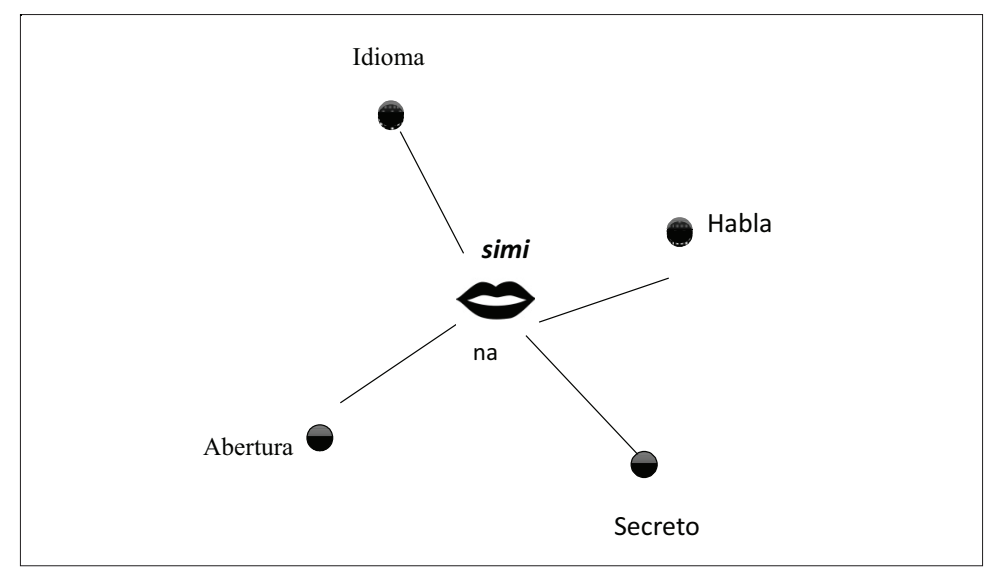

Figura 4. Red semántica del lexema somático simi 'boca'

La red semántica de simi 'boca' expresa cuatro sentidos. Estos son los conceptos de idioma, habla, secreto, y abertura.

\section{Conclusiones}

1. Los lexemas referidos a la anatomía humana del quechuahablante, en general, son términos fuertemente polisémicos. En particular, los lexemas somáticos uma 'cabeza' y simi 'boca' son términos que exhiben una serie de 
conceptualizaciones que se sitúan más allá del significado lingüístico convencional, y permiten reflejar un complejo abanico de posibilidades conceptuales en la lengua y cultura quechua.

2. En el lexema somático uma 'cabeza' se han detectado diez significados periféricos, de los cuales nueve de ellos están constituidos en tres grupos. El primer grupo del eje de verticalidad reúne los sentidos de jefe y parte superior; el segundo grupo de los procesos cognitivos, los conceptos de memoria, pensamiento e inteligencia; y el tercer grupo de la conducta humana, los conceptos de ladrón, sobriedad, cordura y problema. Finalmente, es posible referir el concepto de sentimiento mediante el término uma.

3. En el lexema somático simi 'boca' se han identificado cuatros significados periféricos. Estos son los siguientes: idioma, habla, secreto y abertura. Un hecho particular de este lexema, a diferencia de uma 'cabeza', es que exhibe una cantidad menor de significados. Sin embargo, el número de expresiones figurativas que permiten evidenciar las nuevas conceptualizaciones son mayores que la parte de la biología humana que refiere a la región topográfica superior.

4. La metáfora y la metonimia se presentan como mecanismos que permiten, por un lado, caracterizar la emergencia de las nuevas conceptualizaciones a las que los lexemas somáticos uma 'cabeza' y simi 'boca' están sujetas. Por otro lado, la explicación de la construcción del significado es un proceso, en muchos de los casos presentados, compartidos tanto por la metáfora como por la metonimia. Una vez que las metáforas y las metonimias han sido evocadas por el quechuahablante en la creación de los nuevos conceptos, estos nuevos sentidos llegan a ser esquematizados en la lengua y cultura quechua, con lo cual forman así una red semántica.

\section{Referencias bibliográficas}

Brugman, C. (1981). The Story of 'over': Polysemy, Semantics, and the Structure of the Lexicon. New York: Editorial Garland. 
Evans, V. \& Green, M. (2006). Cognitive Linguistics: An Introduction. Edimburgh: Edimburgh University Press.

Lakoff, G. \& Johnson, M. (1980). Metáforas de la vida cotidiana. Chicago: Chicago University Press.

Lakoff, G. (1998) [1980]. Women, Fire, and Dangerous Things: What Categories Reveal about the Mind. Chicago: University Press.

Langacker, R. (1987). Foundations of Cognitive Grammar: Volume I Theoretical Prerequisites. Standford: Standford University Press.

Torero, A. (2002). Idiomas de los andes. Lingüística e historia. Lima: IFEA.

Tyler, A. \& Evans, V. (2003). The Semantics of English Prepositions: Spatial Scenes, Embodied Meaning, and Cognition. Cambridge: Cambridge University Press. 